\title{
Caracterización del sistema de producción de arroz criollo en La Mojana, Colombia
}

\section{Characterization of the creole-rice production system in La Mojana, Colombia}

\author{
Jorge Cadena Torres ${ }^{1}$ \\ Diana Maria Cuello Pérez ${ }^{2}$ \\ Jorge Luis Romero Ferrer ${ }^{3}$ \\ Shirley Patricia Pérez Cantero ${ }^{4}$
}

DOI: https://doi.org/10.19053/01228420.v18.n2.2021.11610

RESUMEN: La Mojana, es una subregión con paisajes dominados por grandes cuerpos de agua, interconectados por una red de caños y ciénagas. Uno de los sistemas productivos de mayor importancia social es el arroz criollo, al cual se dedican pequeños productores de economía campesina. Dado a que se desconocen aspectos socioeconómicos y técnicos de este sistema, se realizó una caracterización del sistema mediante el método de encuestas estructuradas que captaron aspectos sociales, técnicos y económicos. El método de muestreo usado fue el no probabilístico y los datos se analizaron mediante estadística descriptiva, con medidas de tendencia central y dispersión. Los resultados mostraron que los agricultores de arroz criollo llevan más de 40 años dedicados a esta actividad productiva, en pequeñas áreas de terreno de la finca. Parte de la producción es comercializada en los mercados locales y otra parte es usada para la alimentación del núcleo familiar, por lo que tiene gran importancia en la seguridad alimentaria. A pesar de existir oferta de variedades mejoradas, de mayor rendimiento, buena parte de los agricultores prefieren para su alimentación este tipo de arroz debido a que le atribuyen una mejor calidad culinaria y nutricional. Se identificaron al menos 46 variedades utilizadas para la siembra, la cual se realiza de forma manual con muy baja entrada de insumos de origen químico. Se identificaron las principales limitantes tecnológicas del sistema y se determinaron los costos y rendimientos, lo cual permitió calcular un costo unitario de US\$0,40 $\mathrm{kg}^{-1}$. El principal riesgo que enfrenta este sistema productivo es la pérdida de semillas ancestrales, por lo que se propone la conservación, purificación y escalamiento de las variedades criollas, para devolverlas a la comunidad y así contribuir a la seguridad alimentaria de la población de pequeños productores vinculados a este sistema.

PALABRAS CLAVE: semillas ancestrales, pequeños productores, seguridad alimentaria.
ABSTRACT: La Mojana is a subregion with landscapes dominated by large bodies of water, interconnected by a network of pipes and swamps. One of the productive systems of greater social importance is that of Creole rice, to which small producers of the peasant economy are dedicated. Given that socio-economic and technical aspects of this system are unknown, a characterization of the system was carried out using the structured survey method that captured social, techni$\mathrm{cal}$, and economic aspects. The sampling method used was non-probabilistic and the data were analyzed using descriptive statistics, with measures of central tendency and dispersion. The results showed that Creole rice farmers have been engaged in this productive activity for more than 40 years, in small areas of land on the farm. Part of the production is commercialized in local markets and another part is used to feed the family nucleus, which makes it of great importance in food security. Despite the existence of a supply of improved varieties, with higher yields, a good part of the farmers prefers this type of rice for their food because they attribute a better culinary and nutritional quality to it. At least 46 varieties are used for manually planting, with very low inputs of chemical origin. The main technological limitations of the system were identified and the costs and yields were determined, which allowed calculating a unit cost of US $\$ 0.40 \mathrm{~kg}^{-1}$. The main risk faced by this productive system is the loss of ancestral seeds, which is why the conservation, purification, and scaling of the creole varieties is proposed, to return them to the community and thus contribute to food security.

KEYWORDS: ancestral seeds, small producers, food security.

FECHA DE RECEPCIÓN: 24 de agosto de 2020 FECHA DE ACEPTACIÓN: 26 de mayo de 2021

COMO CITAR ESTE ARTículO: Cadena Torres, J., Cuello Pérez, D., Romero Ferrer, J., \& Pérez Cantero, S. (2021). Caracterización del sistema de producción de arroz criollo en La Mojana, Colombia. Cien. Agri., 18(2): 67-82. https://doi.org/10.19053/01228420.v18.n2.2021.11610

1 Ph.D. Corporación Colombiana de Investigación Agropecuaria-Agrosavia. (Colombia). jcadena@agrosavia.co (D) https://orcid.org/0000-0002-5180-2893

2 IA, Corporación Colombiana de Investigación Agropecuaria-Agrosavia. Turipaná (Colombia).nan_cupe@hotmail.com (iD) https://orcid.org/0000-0002-3979-8908

3 Ph.D. Corporación Colombiana de Investigación Agropecuaria-Agrosavia. Turipaná (Colombia). jromero@agrosavia.co (iD) https://orcid.org/0000-0002-7249-6549

4 M.Sc. Corporación Colombiana de Investigación Agropecuaria-Agrosavia. Turipaná. (Colombia).sperezc@agrosavia.co 


\section{Introducción}

La Mojana es una vasta subregión ubicada al norte de Colombia, que abarca aproximadamente 513464 hectáreas, hace parte de la zona fisiográfica conocida como Depresión Momposina, que está conformada por tierras bajas y cuerpos de agua que cumplen la función de vaso regulador de los ríos más importantes de Colombia (Magdalena, Cauca y San Jorge). El nivel de los cuerpos de agua varía dependiendo de la intensidad de la temporada de lluvias y la dinámica del clima en Colombia, que afecta el volumen de agua de los ríos que vierten sus aguas en la Depresión Momposina (Aguilera, 2004; Urquijo \& Vargas, 2013). La franja más inundable corresponde a la zona norte, en donde confluyen los excesos de agua de lluvias, escorrentías superficiales y excesos de ríos y riachuelos (Terán, et al., 1998; DNP, 2003; Caraballo \& Ossa, 2011; Uribe, 2015). Debido a la suficiente disponibilidad de agua, una de las principales actividades agrícolas en la subregión es el sistema productivo de arroz (Aguilera, 2004; Martínez, 2013).

Este sistema es practicado principalmente por grandes productores con pleno acceso a los factores de producción y semillas mejoradas de alta productividad. En la misma subregión, coexiste un sistema productivo al cual se dedican pequeños productores que conservan la tradición de sembrar pequeñas proporciones de tierra con lo que denominan "arroz criollo." Este tipo de arroz se siembra con variedades muy antiguas que, por sus características, han permanecido en poder de los agricultores, quienes las conservan, multiplican e intercambian. Este sistema se encuentra en alto riesgo debido a los fenómenos extremos del clima, que ha ocasionado grandes inundaciones, perdida de cultivos y semillas. Sobre este sistema productivo, existe poca información y estudios, no se registra en las estadísticas oficiales del cultivo, y en general existe poco conocimiento acerca de su ubicación, variedades, rendimientos, costos, destino de la producción, usos y mercados. Ante el riesgo que enfrenta este sistema productivo, se adelantó el presente estudio, de carácter descriptivo y exploratorio, con el objeto de establecer las características del sistema productivo de arroz criollo en la Subregión de La Mojana en Colombia, como base para proponer alternativas que permitan su conservación y sostenibilidad.

\section{Materiales y Métodos}

El presente trabajo se realizó en la subregión de La Mojana, la cual posee clima tropical, piso térmico cálido, temperatura media de $27,8^{\circ} \mathrm{C}$, precipitación media de $2.846,9 \mathrm{~mm}$ con valores que oscilan entre $1.406,5 \mathrm{~mm}$ en la zona centro-occidental y 4779,7 $\mathrm{mm}$ en el sur oriente. Presenta una época seca entre los meses de enero y marzo (Corpoica, 1998; 2012). 
El marco muestral lo conforma la población de agricultores dedicados al cultivo de arroz criollo en la subregión de La Mojana. En el Censo Nacional Arrocero realizado por Fedearroz en el año 2017 en Colombia (Fedearroz, 2017), no se discriminan las estadísticas en específico para este sistema productivo, sin embargo, en un reporte de Aguilera (2004), se mencionan un total de 9998 agricultores que siembran arroz en un sistema denominado "secano manual". Usando este parámetro como base, se calculó un tamaño de muestra aplicando la fórmula propuesta por Aguilar (2005), para estudios de carácter exploratorio, con una población finita y variables cualitativas:

En donde:

$$
n=\frac{Z^{2} N p q}{p q Z^{2}+(N-1) e^{2}}
$$

Z: Coeficiente para un nivel de confianza del 95\%

p: Proporción de individuos que siembran arroz criollo

q: Proporción de individuos que siembran otro tipo de arroz

e: Error máximo admisible, se asumió en 10\%

Dado a que no se conoce la varianza de la población objetivo, se asumió la mayor varianza posible, considerando los valores de $p=q=0.50$. La aplicación de la anterior ecuación se calculó una muestra (n) de 96 agricultores a encuestar.

Por no contar con el registro actualizado del número de productores dedicados al arroz criollo, se realizó un muestreo no probabilístico en donde los agricultores fueron seleccionados para entrevista acorde a su ubicación sobre las diferentes rutas de acceso. Según el criterio de los técnicos que prestan el servicio de asistencia técnica en las Unidades Municipales de Asistencias Técnica Agropecuaria (UMATA), la producción de arroz criollo se concentra en nueve (9) de los 11 municipios que conforman la subregión de La Mojana. Acorde a la experiencia de los técnicos y las posibilidades de penetración a través de las vías de acceso, se establecieron nueve (9) rutas o recorridos, unas por vía fluvial y otras por vía terrestre. Debido a que al momento de los recorridos se presentaban riesgos de seguridad en el municipio de San Jacinto del Cauca, no se realizaron encuestas en este municipio, lográndose un cubrimiento de ocho municipios y 75 veredas (Tabla I).

Para establecer las características de producción de arroz criollo en la subregión, se diseñó una encuesta estructurada que contempló aspectos sociales, técnicos y económicos, la cual fue aplicada mediante entrevistas presenciales a los agricultores, en su finca o parcela. La encuesta contenía 101 preguntas, con aspectos como: identificación del encuestado y predio, núcleo familiar, educación, características técnicas del cultivo, costos de producción, mercados, comercialización del producto y aspectos financieros del cultivo. 
TABLA I.Numero de encuestas de caracterización aplicada a productores de arroz criollo en ocho municipios de la subregión de La Mojana.

\begin{tabular}{|c|l|l|c|c|c|}
\hline Ruta & Departamento & \multicolumn{1}{|c|}{ Municipio } & No.de veredas & $\begin{array}{c}\text { No. Total } \\
\text { de encuestas }\end{array}$ & $\begin{array}{c}\text { Proporción } \\
\%\end{array}$ \\
\hline 1 & Sucre & San Marcos & 7 & 10 & 9,7 \\
\hline 2 & Sucre & San Benito De Abad & 8 & 8 & 7,7 \\
\hline 3 & Sucre & Majagual & 17 & 23 & 22,3 \\
\hline 4 & Sucre & Guaranda & 9 & 17 & 16,5 \\
\hline 5 & Sucre & Sucre & 11 & 14 & 13,6 \\
\hline 6 & Sucre & Caimito & 8 & 8 & 7,8 \\
\hline 7 & Bolívar & Achí & 9 & 15 & 14,6 \\
\hline 8 & Bolívar & San Jacinto del Cauca ${ }^{\dagger}$ & 0 & 0 & 0,0 \\
\hline 9 & Córdoba & Ayapel & 6 & 8 & 7,8 \\
\hline & Total & & 75 & 103 & 100 \\
\hline
\end{tabular}

tLocalidad con problemas de seguridad.

Para complementar la información de mercados suministrada por agricultores, se realizaron visitas a los molinos procesadores de arroz criollo localizados en la subregión, logrando la aplicación de 70 encuestas. Se incluyeron en esta encuesta aspectos como volúmenes de procesamiento, destino y mercados del del grano, precios y costos de procesamiento. La ubicación de los predios en donde se aplicaron las encuestas a los agricultores y molinos de procesamiento se observa en la Figura 1.

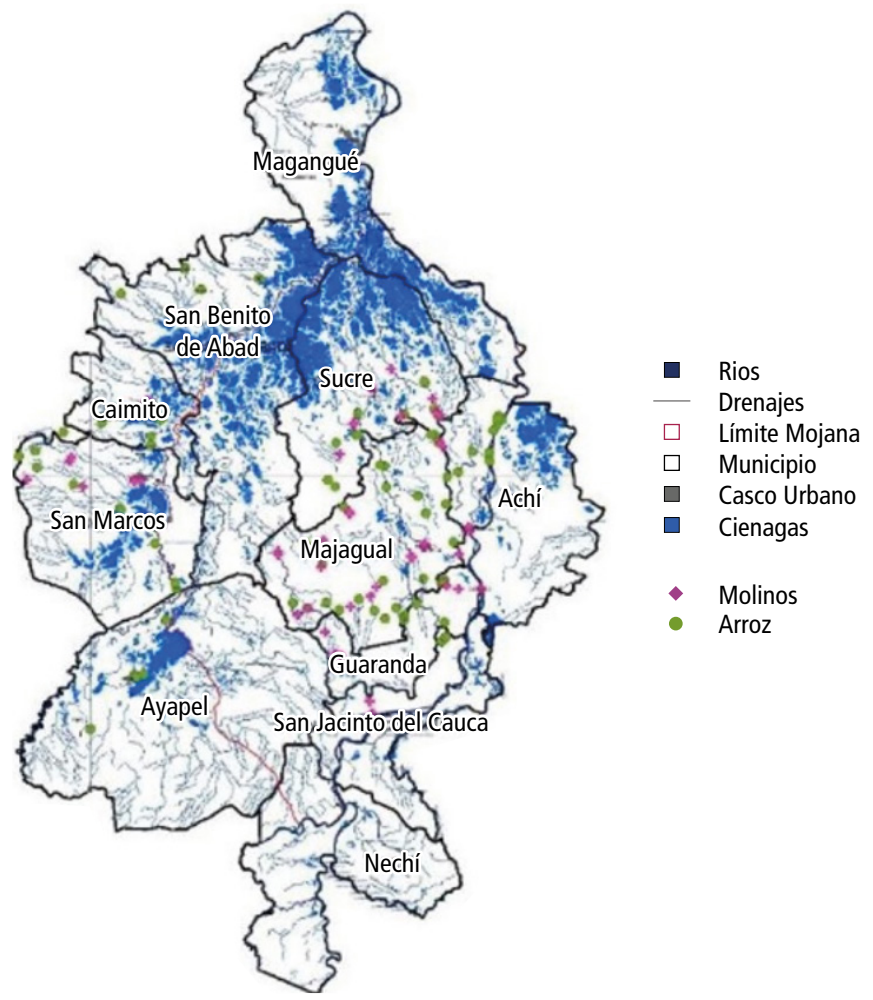

FIGURA 1. Ubicación de los sitios de aplicación de encuestas a agricultores de arroz criollo y molinos en la subregión de La Mojana en Colombia. 
Con la información obtenida de las encuestas se conformó una base de datos en Microsoft Office Excel ${ }^{\circledR}$, lo que permitió el cálculo de parámetros estadísticos de frecuencia, tendencia central y dispersión, usando para ello el programa SAS V9.4 (Cary, NC, USA).

\section{Resultados y Discusión}

\section{Características del sistema de producción}

Los resultados mostraron que la mayor parte de agricultores en la subregión de La Mojana llevan más de 40 años dedicados a la producción de arroz criollo, actividad que ha sido tradicional y para la siembra usan variedades o semillas de tipo criollo que conservan, comparten e intercambian, desde hace muchos años. El núcleo familiar de los cultivadores está conformado por el esposo, la esposa y un promedio 4,2 hijos por familia, esto último con un rango amplio de variación (0 a 15 hijos por familia y $C V=63,6 \%$ ). A la siembra y cultivo del arroz criollo se dedican principalmente los varones de cada familia, aunque también participan en las actividades de campo el núcleo familiar completo, algunos vecinos y familiares, en forma colaborativa.

La unión marital que predomina es unión libre, seguida de casados, solteros y viudos. El nivel de educación predominante es analfabeto $(34,9 \%)$, aunque existen agricultores con algún grado de educación, principalmente a nivel de básica primaria, secundaria, técnicos y muy pocos profesionales (Figura 2). Se resalta que buena parte de los agricultores reportan la educación como incompleta (44,7\%), lo cual muestra un alto grado de deserción escolar. Estas cifras de analfabetismo contrastan con las reportadas en el último Censo Nacional Agropecuario, en donde se indica que el analfabetismo en

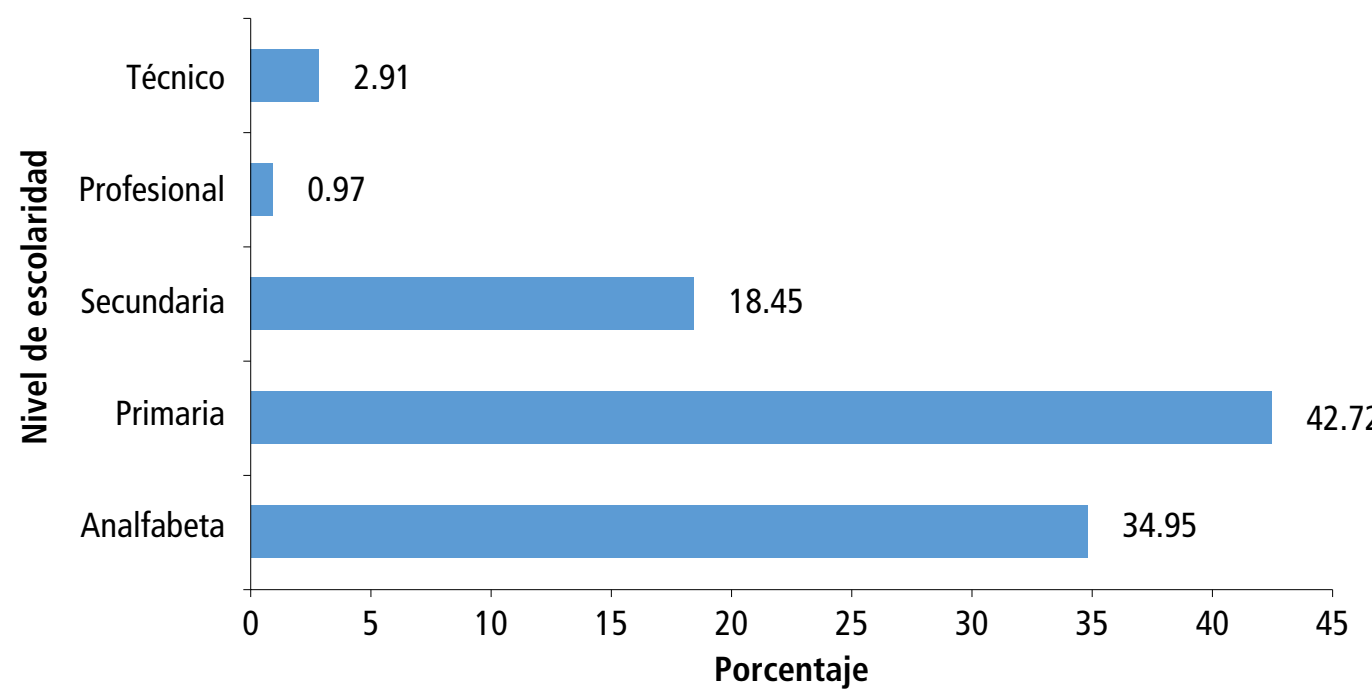

FIGURA 2. Nivel de Escolaridad de los agricultores vinculados a la producción de arroz criollo en la subregión de La Mojana. 
la población rural dispersa mayor de 15,0 años, en Colombia, es del 12,5\% (DANE, 2016). El analfabetismo es un fenómeno que afecta en especial a la Región Caribe de Colombia, en donde las zonas rurales son las más afectadas, principalmente por deficiente cobertura, calidad, permanencia o estabilidad en la prestación del servicio.

El promedio de edad de los agricultores es 51,5 años, la tenencia de la tierra es principalmente propia $(65,0 \%)$, y el área total de los predios fluctúa entre 0,3 y 16,5 hectáreas. Del área total, los agricultores destinan a la producción de arroz criollo pequeñas áreas de su finca o parcela, que fluctúan entre 0,1- 8,0 ha, dado que el resto del terreno es dedicado a la siembra de otros cultivos, entre los que se encuentran el arroz de tipo mejorado, yuca, maíz, pasturas y hortalizas. Esta pequeña área de siembra de arroz criollo es calculada anualmente con base en las necesidades de grano para el consumo familiar.

Para su alimentación, los agricultores prefieren el consumo de las variedades criollas de arroz, debido a que les atribuyen características culinarias y nutricionales superiores que las de los arroces mejorados. Entre las características de calidad que más se resaltan en el arroz criollo se mencionan el sabor, aroma, cocción, apertura del grano, rendimiento, tamaño y soltura. Estos resultados resaltan la importancia que tiene el arroz criollo como componente de la dieta familiar y la seguridad alimentaria de los pequeños productores rurales de la subregión de La Mojana, en especial debido a que es la base fundamental de la dieta diaria del agricultor y su familia. De igual forma se resalta la valoración que hacen los agricultores a la calidad culinaria de las variedades criollas pues, aunque tienen acceso a otros tipos de grano, para alimentar a su núcleo familiar, prefieren las variedades de tipo criollo.

Las fincas en su gran mayoría presentan una gran dificultad de acceso debido a la fluctuación de los niveles del agua en los caños, ciénagas y cuerpos de agua, lo cual deteriora las vías de acceso. El acceso a las fincas es principalmente por vía terrestre, a través de caminos, carreteras y trochas, aunque también existen fincas a las cuales la única vía de acceso es fluvial, y otras en parte terrestre y en parte fluvial.

La principal ocupación de los encuestados es la agricultura, aunque existe una alta proporción de entrevistados que trabajan como jornaleros en otras fincas o parcelas vecinas, en actividades de ganadería y/o agricultura, como también pescadores, artesanos, ebanistas y moto taxistas. Este último es un servicio de transporte público informal, al cual se dedican muchos habitantes de las zonas rurales de Colombia, debido a la crisis en el sector agropecuario, pues encuentran en esta actividad una forma para generar ingresos para poder sostener a sus familias. El mototaxismo ha sido reconocido como un fenómeno social de grandes proporciones que afecta en especial a las zonas rurales, al privarla de la mano de obra necesaria para las labores de campo. 
Muchas personas, especialmente jóvenes hijos de agricultores y jornaleros, están emigrando a los centros urbanos en búsqueda de mejores oportunidades, y al no encontrar otras fuentes de empleo se dedican de forma temporal o permanente a prestar este servicio de transporte informal (Sánchez, 2011).

En cuanto a las variedades usadas para la siembra, en total se lograron referenciar alrededor de 46 variedades de arroz criollo, con distinta cantidad de nombres vernáculos, en donde las más frecuentes o preferidos por los productores fueron Guayaquil, Chilimico, Ina Mono, Ligerito Mono, Belepan Blanco y Campeón (Figura 3).

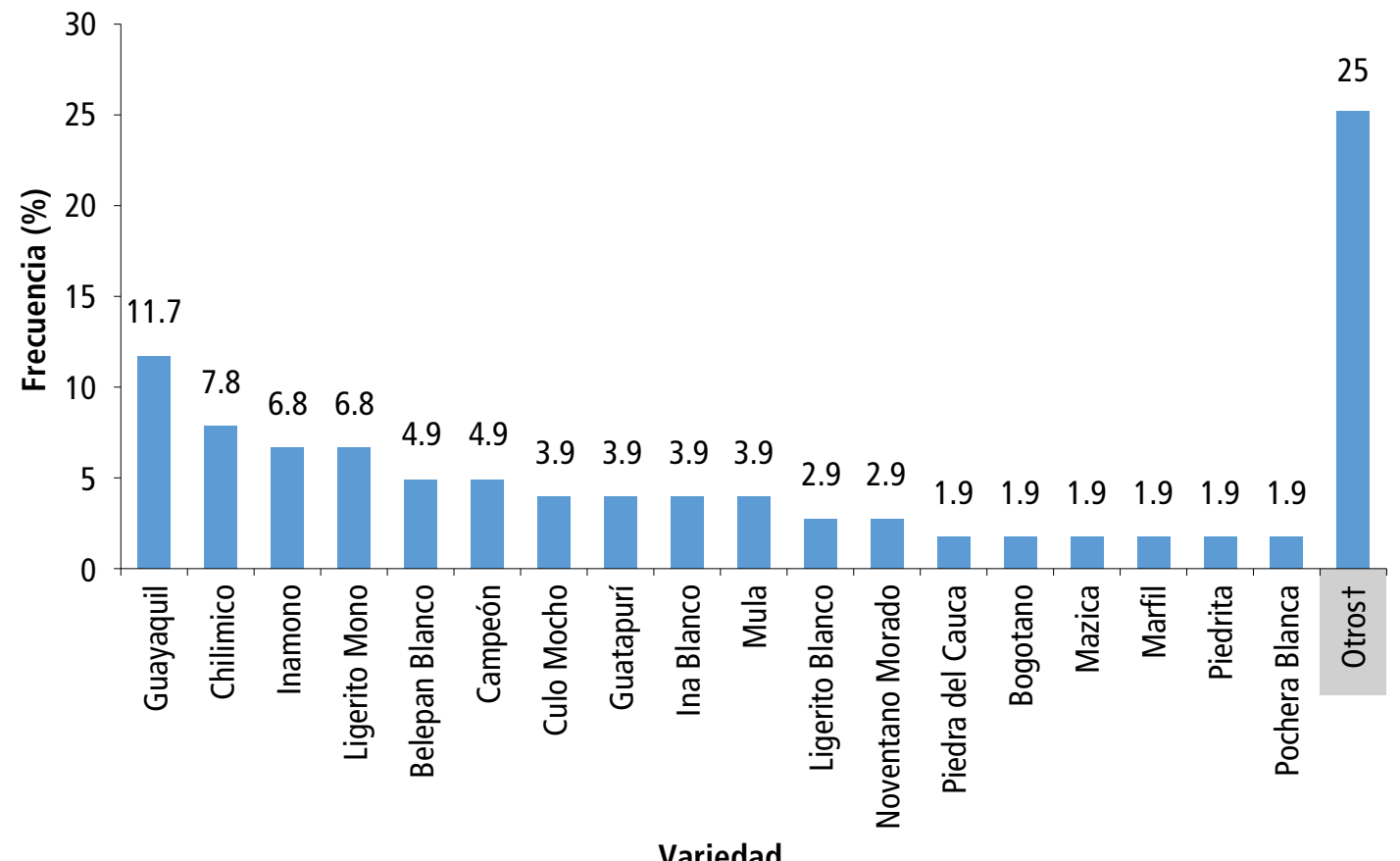

FIGURA 3. Variedades de arroz criollo sembradas en la subregión de La Mojana, otras variedades: Belepan moro, Blubonet, Caribe Blanco, Carretero, Cheverito Blanco, Cheverito Ligerito, Chilimico Ligerito, Chucha Larga, Culo Mocho, Fortuna, Guayaquil Mono, Liberal, Marfil Morado, Maria La Baja, Mico, Mula - Campeón, Mula- Chilimico, Mulita Blanco, Nocentano Mono, Palmira, Pedro Costal, Pedro Seco, Perla, Pochera Sucia, Pollera Sucia y Tumba la Cama.

La semilla para la siembra es obtenida de sus propios cultivos, vecinos u otros agricultores, para lo cual anualmente reservan parte de la cosecha para usarla como semilla en la siguiente temporada de siembras. La semilla es conservada colocando mazos de arroz con espigas, colgados en el cobertizo de la vivienda. Una alta proporción de los agricultores mencionaron la perdida de sus semillas debido a las crecientes de los ríos que han ocurrido en los últimos años en Colombia, lo cual ha obligado al desplazamiento periódico de la población hacia zonas más altas y secas. Esto hace de las variedades criollas un recurso muy vulnerable a la perdida, tanto por las inundaciones, como al ataque de plagas y enfermedades en el cobertizo de las viviendas. No existe al respecto un programa gubernamental de fomento y multiplicación de la 
semilla de variedades criollas, lo que resalta la importancia de su colecta y conservación en los bancos generales de la nación. Cuando ocurren pérdidas de la semilla, los agricultores recurren a un vecino y unos pocos a los molinos de procesamiento. Dado este intercambio de semilla, es muy probable que la identidad genética de muchas de estas variedades se haya perdido, por lo que se hace necesaria su colecta, purificación y caracterización.

El sistema de siembra utilizado es principalmente manual, practicado por el $94,4 \%$ de los agricultores (Figura 4). La mayor parte realizan esta labor con la ayuda de un espeque de madera o chuzo, con el cual perforan el suelo y depositan la semilla directamente en el terreno. Otros realizan la siembra manual al voleo, arrojando manualmente la semilla sobre el terreno húmedo. Otro pequeño porcentaje de agricultores realizan un semillero para luego de 30-45 días, trasplantar en forma manual las plántulas al sitio definitivo. Muy pocos agricultores realizan siembra mecánica $(5,8 \%)$, pues es un recurso poco disponible para áreas tan pequeñas de siembra. Para la siembra se utiliza una gran variación en la cantidad de semilla, entre 25 y $70 \mathrm{~kg} \mathrm{ha}^{-1}$, siendo las menores cantidades usadas cuando el sistema de siembra es manual a chuzo. En promedio se usa un total de $51,7 \mathrm{~kg} \mathrm{ha}^{-1}$ de semilla. Esta cantidad resulta especialmente alta, considerando que la mayor parte de los agricultores realizan la siembra en forma manual. Se presenta aquí una oportunidad de tecnificación y mejora para los programas de fomento del cultivo, en especial para la implementación de prácticas de siembra con implementos diseñados para la agricultora a pequeña escala.

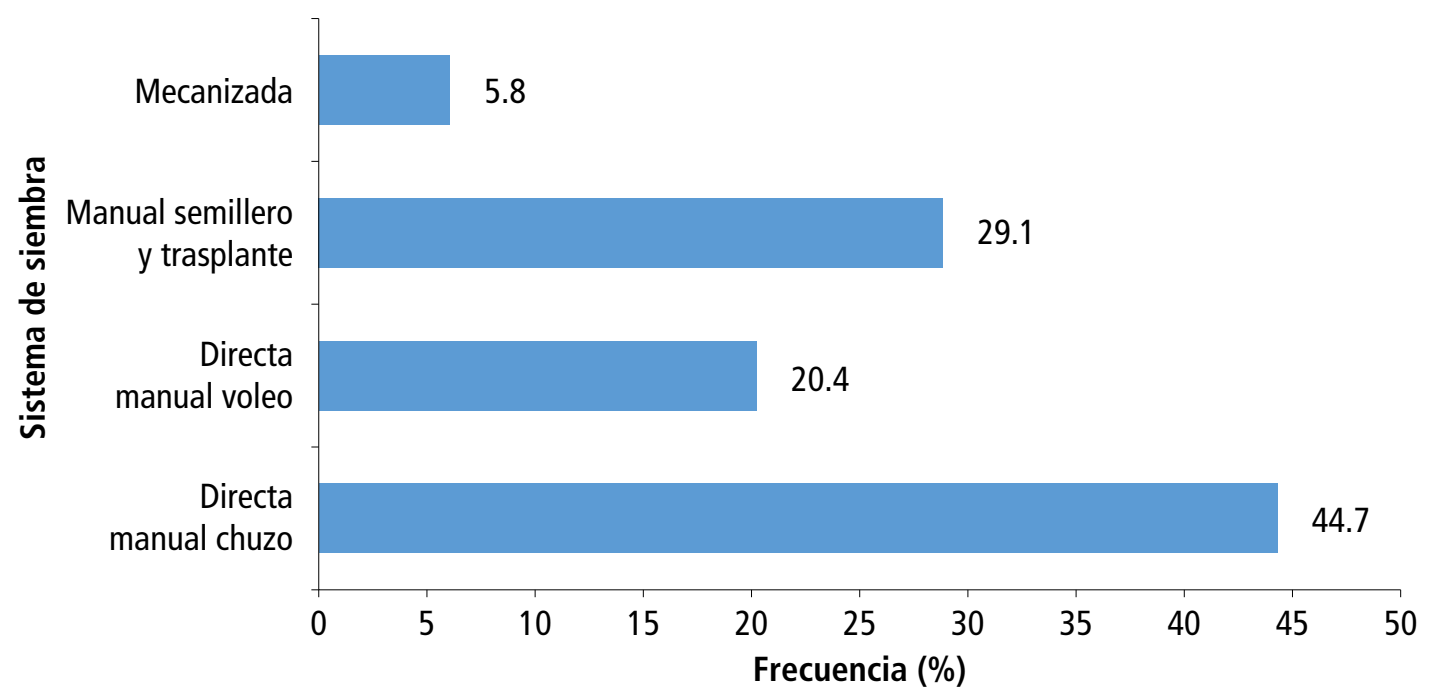

FIGURA 4. Sistemas de siembra utilizados por los agricultores para la siembra de arroz criollo en la subregión de La Mojana.

La siembra se realiza en dos oportunidades durante el año (primer y segundo semestre agrícola). Las siembras de primer semestre se realizan entre los meses de marzo y mayo, con el inicio de las lluvias, mientras que las 
siembras de segundo semestre se realizan entre junio y septiembre. El ciclo del cultivo es muy variable y se extiende por periodos entre 90 y 180 días, dependiendo de la variedad utilizada. Los agricultores disponen de variedades precoces, las cuales reciben el apelativo de arroces "Ligeritos" o "Noventanos," y presentan ciclos de cultivo entre 90-110 días. Las variedades más tardías presentan ciclos de cultivo entre 120-130 días. El ciclo del cultivo también varía con la época de siembra, siendo más precoces los arroces sembrados en el primer semestre del año (113-119 días), que los sembrados en el segundo semestre (114-150 días). Con lo anterior la cosecha de arroz criollo ocurre en dos temporadas, la primera entre los meses de junio y agosto, correspondiente a las siembras de primer semestre, $y$ entre octubre $y$ diciembre, correspondiente a las siembras de segundo semestre.

Con relación al uso de insumos, en general el cultivo de arroz criollo se realiza con la mayor parte de las actividades realizadas en forma manual y un bajo uso de insumos de origen químico. La labor de control de malezas es de las pocas que obligan el uso de productos de origen químico, donde el 88.3\% recurren al uso de herbicidas para su control, y por tanto utilizan una gran variedad de productos entre los que se encuentran aminas, glifosato y propanil, entre los más comunes.

Históricamente, los agricultores de arroz criollo en La Mojana no realizaban ningún tipo de fertilización a los cultivos, por la fertilidad natural de los suelos (Camacho, 2017). Esto ha cambiado en los últimos años y cada vez más recurren al uso de insumos de origen químico. En este trabajo se encontró que aproximadamente la mitad de los agricultores aún no aplica fertilizantes edáficos al cultivo de arroz $(51,4 \%)$, mientas la otra mitad $(46,6 \%)$, aplican urea en cantidades que oscilan entre 1 y 2 bultos de $50 \mathrm{~kg} \mathrm{ha}^{-1}$ y una pequeña proporción (0.9\%) aplican la urea en combinación con el fertilizante triple-15 (bultos por $50 \mathrm{~kg}$ ), en dosis de 1,0+1,0 bultos ha-1. La aplicación de los fertilizantes edáficos se realiza en forma manual. Algunos agricultores (13,5\%), aplican fertilizantes foliares, para lo cual utilizan principalmente el sulfato de amonio en dosis de $1 \mathrm{~kg} \mathrm{ha-1.}$

Para el control de enfermedades, la mayor parte de los productores $(81,6 \%)$ manifestó no usar ningún tipo de fungicidas, mientras que el restante 18,4\% manifestó usar productos de origen químico. Los principales problemas que afectan el cultivo de arroz criollo son la Pyricularia y Rhizoctonia. La primera es causada por el hongo Pyricularia oryzae Cav que afecta las hojas e inflorescencias de la planta de arroz. La segunda se conoce comúnmente como añublo del arroz y es causado por el hongo Rhizoctonia solani Kuhn y afecta principalmente las hojas. El control de insectos plagas, es la segunda labor, después de control de malezas, que más obliga el uso de productos de origen químico, pues una proporción alta de los agricultores manifiestan usar insecticidas de origen químico para su control (76,7\%). Para lo anterior usan una gran variedad de productos, en donde la más frecuente es el uso de la 
cipermetrina. El 23,3\% de los agricultores no aplican ningún tipo de productos para el control de insectos plagas.

La cosecha se realiza preferencialmente en forma manual y el arroz cosechado es principalmente "trillado" a mano, proceso mediante el cual se separa el grano del resto de material vegetal (raquis y espiga), y el grano resultante denominado "arroz paddy," aun con cascara es empacado en sacos para su transporte a los sitios de acopio o procesamiento. El saco preferido para empacar es el de fibra natural de fique, aunque también se usan sacos plásticos, bolsas, empaques de fertilizantes o cualquier otro tipo de empaque que tengan disponible en la finca. El peso del saco de arroz paddy verde empacado varía entre 45,0 y 80,0 kilogramos, siendo más frecuente los sacos con capacidad de 70,0 kg de peso.

Los rendimientos obtenidos en la última cosecha fueron en promedio de $2.253,6 \mathrm{~kg} \mathrm{ha}^{-1}$ con una muy alta variación entre los reportes de las fincas $(C V=52 \%$, D. Estd=1.181,1 kg ha-1). La anterior variación fue ocasionada por el comportamiento errático de la temporada de lluvias, registrándose un 17,5\% de agricultores con pérdidas totales del cultivo, hasta agricultores con rendimientos máximos de $4.900,0 \mathrm{~kg} \mathrm{ha}^{-1}$. Lo anterior, aunque indica una alta potencialidad de este cultivo, también es el reflejo de la alta vulnerabilidad de los productores a las variaciones de las condiciones climáticas, principalmente al comportamiento de la temporada de lluvias. De igual forma, la alta variación en los rendimientos puede ser ocasionada por los distintos niveles tecnológicos aplicados por los agricultores en el cultivo de arroz criollo, pues la asistencia técnica para el cultivo es escasa, representada principalmente por los técnicos de las UMATA y algunos técnicos de la Federación Nacional de Arroceros de Colombia (Fedearroz) que hacen presencia en la zona.

Con relación al destino de la producción, un poco más de la mitad (57,5\%), de la producción del arroz criollo se destina al autoconsumo, resaltando la importancia de este cultivo desde el punto de vista de la seguridad alimentaria para los pobladores de la subregión. La cantidad restante es destinada a la venta comercial, reservada como semilla para la siguiente cosecha, usada para el trueque por otros productos de la canasta familiar o utilizada para el pago de algunos jornales utilizados en el manejo del cultivo. Esto último ocurre debido a la falta de recursos para pagar los jornales del cultivo, con lo cual algunos agricultores solicitan a otros "donar" su jornada de trabajo, bajo la promesa de retribuir al final esos jornales, con la producción de arroz. El arroz que se destina para semilla y autoconsumo es almacenado aún con la espiga, en el cobertizo de la vivienda del agricultor, de la cual se va retirando diariamente una cantidad denominada "puño" o "mazo", de acuerdo con el consumo diario de la familia. Este arroz es procesado en la misma finca o en fincas cercanas, mediante el uso de pilones de madera. 
El arroz que es destinado a la comercialización es transportado en sacos a los sitios de comercialización o molinos ubicados en las veredas y cabeceras municipales cercanas. Para el transporte del arroz desde la finca o parcela a los molinos o sitios de comercialización se utiliza todo tipo de medios de transporte, desde canoas, lanchas, carros, animales de carga, tractores y motos, siendo este último el sistema de transporte más común. Las distancias al centro de comercialización varían entre 1,0 y 50,0 km.

El grano de arroz criollo es muy apetecido en la subregión por los consumidores finales, dadas las cualidades culinarias que se le reconocen, lo cual hace que en la subregión lo clasifiquen como "arroz fino". Por lo anterior, no se presentan problemas de comercialización para este tipo de arroz. Durante la encuesta se detectaron 34 sitios de comercialización, en nueve municipios de la subregión, siendo el más importante el municipio de Majagual, con un mayor número de sitios de acopio o compra.

El precio de comercialización del arroz criollo es en promedio superior al de otros tipos de arroz que se comercializan en la subregión. El arroz proveniente de los cultivos extensivos y tecnificados recibe el nombre de "Federado" en razón a que las variedades sembradas son producidas por el Programa de Mejoramiento Genético de la Federación Nacional de Arroceros (Fedearroz). Para la época de la encuesta el arroz paddy o con cascara del tipo "Federado" en el mercado local se comercializaba a un precio de $\$ 946,4 \mathrm{~kg}^{-1}$, mientras que el arroz criollo era comercializado a un precio de $\$ 1.283 \mathrm{~kg}^{-1}$ (Fedearroz, 2018). Lo anterior representa un precio $35 \%$ superior del arroz criollo, lo cual se atribuye al aprecio y alta demanda, debido a las características de calidad que se le reconocen.

El precio del arroz en el mercado local presenta una variación a través del año, siendo mayo, junio y julio los meses de precios más altos, debido a la poca oferta disponible, y agosto, octubre y diciembre los meses de precios más bajos. El precio de compra es fijado entre el vendedor y comprador, de acuerdo con la oferta del producto en el mercado, y para la fijación de este se tienen en cuenta características como época del año, humedad, impurezas, color y tamaño del grano. Los agricultores mencionan en su mayoría no tener ningún tipo de problemas para la comercialización del arroz criollo, excepto por los bajos precios en algunas temporadas del año (agosto a diciembre) y algunos monopolios que se intentan establecer para la comercialización de este tipo de arroz.

Entre las restricciones tecnológicas a la producción la que los productores identifican como de mayor importancia es la poca disponibilidad de semilla para la siembra, debido a los efectos de las inundaciones han ocasionado la perdida de muchos cultivos y la consecuente pérdida de las semillas de las variedades, pues éstas no son objeto de ningún programa de fomento o multiplicación por parte de entidades del estado. Esto se convierte en la principal 
limitante tecnológica identificada para la ampliación de las áreas y la siembra de los cultivos, por lo que proyectos futuros deben considerar el establecimiento de un programa local de fomento y producción de semillas de estas variedades. De otro lado, dado el comercio informal de semilla, es muy probable que la identidad genética de las variedades se haya deteriorado.

\section{Molinos procesadores}

Se entrevistaron 70 propietarios y administradores de molinos que procesan arroz criollo y otros tipos de arroz producidos en la subregión. La capacidad de procesamiento de la mayoría de los molinos está entre 300 y $400 \mathrm{~kg} \mathrm{día}^{-1}$ que utilizan como fuente propulsora la energía eléctrica y una menor proporción el combustible tipo Diesel. La cantidad de arroz paddy procesado en el último año en los molinos fue de 4.569 t, de las cuales correspondieron a arroz criollo 1.514 t (33,1\% del arroz procesado). El período de mayores volúmenes de procesamiento de arroz fue enero a marzo. La cantidad procesada disminuye a mediados de año y aumenta en los meses de octubre a diciembre.

El arroz procesado es empacado como arroz blanco en sacos de 45 a $50 \mathrm{~kg}$ y comercializado en los mercados de ciudades como Guaranda, Majagual, San Marcos y Sucre. Otros sitios de comercialización son las tiendas ubicadas en veredas aledañas a los molinos y las tiendas y graneros ubicados en las veredas donde se ubican los cultivos. En los graneros, el arroz criollo es reempacado en bolsas de $250 \mathrm{~g}$, las cuales son comercializadas entre los consumidores finales.

\section{Costos de producción}

Para determinar el patrón de costos del arroz criollo en la subregión de La Mojana se organizaron en forma secuencial las actividades del cultivo y las labores que se realizan para que cada agricultor individualmente indicara la cantidad de mano de obra utilizada, valor de la mano de obra, insumos y su valor. Los resultados se presentan como un promedio de las 103 encuestas realizadas, e indicaron que el costo total de producción de arroz criollo en la subregión fue de $\$ 2.682 .073,3 \mathrm{ha}^{-1}$, equivalente a US $\$ 907,2 \mathrm{ha}^{-1}$ siendo la mano de obra, el componente más importante, con una participación del $58 \%$ de los costos totales de producción. Las labores que más requieren el uso de mano de obra son en su orden la preparación manual de suelos, la siembra y la cosecha (Tabla II). 
TABLA II.Costos de producción de arroz criollo en la subregión de La Mojana (US\$ ha-1)†.

\begin{tabular}{|c|c|c|c|c|}
\hline Ítem de costos & $\begin{array}{l}\text { No. de } \\
\text { Jornales }\end{array}$ & $\begin{array}{c}\text { Valor del Jornal } \\
\text { US\$ }\end{array}$ & $\begin{array}{l}\text { Valor Total } \\
\text { US\$ }\end{array}$ & $\begin{array}{c}\text { Participación } \\
\%\end{array}$ \\
\hline Preparación de Semilleros & 1,9 & 9,3 & 17,7 & 2,0 \\
\hline Preparación de suelos & 9,0 & 10,2 & 91,8 & 10,1 \\
\hline Siembra & 10,1 & 9,8 & 99,0 & 11 \\
\hline Resiembra & 0,6 & 9,0 & 5,4 & 0,6 \\
\hline Deshierba manual & 5,0 & 9,7 & 48,5 & 5,4 \\
\hline Fertilización & 0,8 & 10,1 & 8,1 & 0,9 \\
\hline Control de Enfermedades & 0,3 & 10,8 & 3,2 & 0,4 \\
\hline Control de Plagas & 1,4 & 11,4 & 16,0 & 1,7 \\
\hline Control de Malezas & 1,3 & 11,3 & 14,7 & 1,6 \\
\hline Cosecha & 31,3 & 7 & 219,1 & 24,3 \\
\hline Subtotal mano de obra & 61,7 & 9,9 & 523,4 & 58,0 \\
\hline Fertilizantes edáficos (Bultos) & 0,7 & 23,6 & 16,5 & 1,7 \\
\hline Fertilizantes foliares ( $\mathrm{kg} \circ \mathrm{L}$ ) & 2,8 & 8,3 & 23,2 & 2,6 \\
\hline Fungicidas $(\mathrm{kg} \circ \mathrm{L})$ & 0,4 & 12,8 & 5,1 & 0,5 \\
\hline Herbicidas (kg o L) & 3,3 & 10 & 33,0 & 2,8 \\
\hline Insecticidas (kg o L) & 0,7 & 14,7 & 10,3 & 1,0 \\
\hline Subtotal Insumos & 7,9 & 13,9 & 88,2 & 8,6 \\
\hline Arrendamiento & & 45,2 & 45,2 & 5,0 \\
\hline Asistencia Técnica & & 0,0 & 0,0 & 0,0 \\
\hline Maquinaria & & 42,1 & 42,1 & 4,6 \\
\hline Riegos & & 0,0 & 0,0 & 0,0 \\
\hline Drenajes & & 0,0 & 0,0 & 0,0 \\
\hline Empaques & & 66,2 & 66,2 & 7,3 \\
\hline Transporte & & 68,2 & 68,2 & 7,5 \\
\hline Trilla & & 40,3 & 40,3 & 4,4 \\
\hline Pajareo & & 1,5 & 1,5 & 0,2 \\
\hline Secado & & 12,1 & 12,1 & 1,3 \\
\hline Pilado & & 7,3 & 7,3 & 0,8 \\
\hline Otros & & 12,7 & 12,7 & 1,4 \\
\hline Subtotal & & & 295,6 & 32,5 \\
\hline Costo Total & & & $\$ 907,2$ & 100,0 \\
\hline Rendimiento & & & 2253.6 & \\
\hline Costo por kilogramo & & & $\$ 0.4$ & \\
\hline $\begin{array}{l}\text { Precio de venta arroz paddy } \\
\text { por kilogramo }\end{array}$ & & & 0.434 & \\
\hline Ingresos brutos & & & 978.1 & \\
\hline Ingresos netos & & & 70.9 & \\
\hline Rentabilidad neta & & & $7.8 \%$ & \\
\hline
\end{tabular}

†Promedio de 103 encuestas realizadas. TRM utilizada para el cálculo \$2.956.43. 
Dado el bajo uso de productos de origen químico, el costo de los insumos equivale tan solo a un $8,6 \%$ de los costos totales. En este rubro, los componentes más importantes son los fertilizantes edáficos, foliares y los herbicidas. Otros costos de producción en el sistema de producción están representados por el arrendamiento de tierra, uso de maquinaria agrícola, empaques y transporte del producto cosechado a los sitios de procesamiento y comercialización, lo que representan el 32,5\% de los costos totales. De estos últimos, el componente más importante es el transporte a los centros de comercialización, seguido de empaques, trilla o procesamiento, maquinaria y arrendamiento de tierra.

Teniendo en cuanta el promedio de rendimiento reportado por los agricultores para la última cosecha (2253,6 $\left.\mathrm{kg} \mathrm{ha}^{-1}\right)$, lo anterior arroja un costo unitario de US\$0,40 $\mathrm{kg}^{-1}$, lo cual lo hace medianamente competitivo en el mercado del grano. Por su parte, la rentabilidad total del arroz criollo fue muy baja $(7,8 \%)$, lo que indica que por cada unidad monetaria invertida en el costo se recupera y genera adicionalmente tan solo 7,8 centavos e indica que la producción se encuentra muy cerca del punto de equilibrio, sin dejar mayores excedentes para los productores.

\section{Conclusiones}

Los anteriores resultados permiten concluir que el cultivo de arroz criollo es un sistema de alta importancia social, para la seguridad alimentaria de los pequeños productores de la subregión de La Mojana. Por ser un sistema manejado enteramente de forma manual y con una baja entrada de insumos de origen químico, es un sistema que fácilmente podría evolucionar a la producción con Sello Verde, introduciéndole algunas tecnologías de substitución de insumos, que ya han sido desarrolladas para otros cultivos. Para mejorar los ingresos económicos de los productores podría aprovecharse la calidad culinaria para generar mayor valor agregado, buscando la ubicación de este tipo de arroz en los estándares de consumo como "arroz fino" lo cual le confiere un alto potencial para el mercado de arroces especiales en supermercados y restaurantes de alta gama. Las variedades de arroz criollo están en riesgo de desaparecer debido a las frecuentes inundaciones y perdida de los cultivos, por lo que se hace necesario su conservación, purificación y devolución a las comunidades de pequeños productores, a fin de contribuir con la seguridad alimentaria de la población rural. A este respecto se recomienda la implementación de núcleos productivos de arroz fino, con plantas de procesamiento, clasificación y empaque de un producto con valor agregado.

\section{Agradecimientos}

Este estudio fue financiado mediante el Convenio No. 002 del 1 de febrero de 2012, entre el Fondo de Adaptación y la Corporación Colombiana de 
Investigación Agropecuaria (Agrosavia), del gobierno de Colombia, a quien los autores expresan sus agradecimientos. Código de Agenda 1774.

\section{Referencias}

Aguilar, S. (2005). Fórmulas para el cálculo de la muestra en investigaciones de salud. Salud en Tabasco 11 1 1-2):333-338. Disponible en: https://www.redalyc. org/pdf/487/48711206.pdf

Aguilera, M. (2004). La Mojana: riqueza natural y potencial económico. Banco de La República: Documentos sobre Economía Regional, Centro de Estudios Regionales-CER, Documento No. 48. Cartagena de Indias, octubre de 2004. 73 p. http://repositorio.banrep.gov.co/bitstream/handle/20.500.12134/3204/ La\%20Mojana.\%20Riqueza\%20natural\%20y\%20potencial\%20econ\%C3\%B3mico. pdf? sequence $=1$ \&isAllowed $=y$

Camacho, J. (2017). Acumulación tóxica y despojo agroalimentario en La Mojana, Caribe colombiano. Revista Colombiana de antropología 53(1):123-150. http:// www.redalyc.org/articulo.oa?id $=105050684005$

Caraballo, P. \& Ossa, VJ. (2011). Inundaciones en la mojana: ¿via crusis social o condición ambiental?. Revista Colombiana de Ciencia Animal Recia 3(1):198210. Disponible en: https://revistas.unisucre.edu.co/index.php/recia/article/ download/362/404

CORPOICA. (1998). Caracterización biofísica, socioeconómica y técnica de los sistemas de producción agropecuarios de la subregión de La Mojana: Sistemas de producción mixtos. Informe Final del Convenio Corpoica-INAT, Cereté, diciembre de 1998. Corporación Colombiana de Investigación Agropecuaria, Corpoica, Regional 2. Cereté, Córdoba. 57 p.

CORPOICA. (2012). Actualización de la información socioeconómica y climática de la Región de La Mojana. Informe Final de Proyecto. Corporación Colombiana de Investigación Agropecuaria, Corpoica, Regional 2. Cereté, Córdoba 132 p.

DANE. (2016). Tercer Censo Nacional Agropecuario: Tomo 2: Resultados. DANE-Ministerio de Agricultura y Desarrollo Rural, Bogotá, Colombia, noviembre de 2016. 1035 p. Disponible en https://www.dane.gov.co/files/images/foros/ foro-de-entrega-de-resultados-y-cierre-3-censo-nacional-agropecuario/CNATomo2-Resultados.pdf

DNP. (2003). Programa de Desarrollo Sostenible de la región de La Mojana (PDSM). Departamento Nacional de Planeación y Organización de las Naciones Unidas para la Agricultura y la Alimentación FAO. Bogotá, Colombia. 567 p.

Federación Nacional De Arroceros-Fedearroz. (2017). IV Censo Nacional Arrocero. Federación Nacional De Arroceros, Fondo Nacional del Arroz, División de Investigaciones Económicas. Bogotá D. C., Mayo de 2017, Colombia. Disponible en http://www.fedearroz.com.co/doc_economia/Libro\%20Censo\%20General.pdf

Federación Nacional De Arroceros-Fedearroz. (2018). Precio promedio mensual del arroz paddy verde en Colombia. Bogotá, Colombia. [Fecha de consulta: septiembre de 2018]. Disponible vía Dialnet http://www.fedearroz.com.co/new/ precios.php

Martínez, A. (2013). Caracterización socioeconómica de los sistemas de producción de la región de La Mojana en el Caribe de Colombia. Corpoica Ciencia y 
Tecnología Agropecuaria 14(2): 165-185. Disponible en http://revista.corpoica. org.co/index.php/revista/article/download/406/322

Sánchez, A. (2011). La economía del mototaxismo: el caso de Sincelejo. Banco de la República, Documentos de Trabajo sobre Economía Regional. No. 4, Cartagena, Marzo de 2011.54 p.Disponible en http://repositorio.banrep.gov.co/bitstream/ handle/20.500.12134/3132/La\%20econom\%C3\%ADa\%20del\%20mototaxismo.\%20El\%20caso\%20de\%20Sincelejo.pdf?sequence=1 \&isAllowed=y

Terán, C; Jimenez, C; Conzález, C \& Villaneda, E. (1998). Metodología para la zonificación agroclimática de la región de La Mojana mediante el sistema de información geográfica ARC/Info. Corpoica Ciencia y Tecnología Agropecuaria 2(2): 19-26. Disponible via Dialnet https://dialnet.unirioja.es/servlet/ articulo?codigo $=5624812$

Uribe, MR. (2015). Caracterización de la dinámica de inundación en la región de La Mojana y las estrategias implementadas para mitigar sus consecuencias sociales en el período comprendido entre los años 2001 y 2012. Trabajo de grado. Bogotá, Colombia. Pontificia Universidad Javeriana, Facultad de Estudios Ambientales y Rurales, Carrera de Ecología. 79 p. Disponible en https://repository.javeriana. edu.co/handle/10554/12448

Urquijo C \& Vargas M. (2013). Caracterización territorial y de inundaciones en la región de La Mojana. Trabajo De Grado. Bogotá, Colombia, Universidad Católica de Colombia, Facultad de Ingeniería, Programa de Ingeniería Civil. 90 p. Disponible en https://repository.ucatolica.edu.co/bitstream/10983/911/2/Caracterizaci\%C3\%B3n-territorial-inundaciones-regi\%C3\%B3n-La\%20Mojana.pdf 